\title{
Energies renouvelables : l'un des pivots du développement durable en Algérie
}

\author{
Wassila Iguergazix Dahmoun
}

\section{Universite de sherbooke}

\section{Résumé :}

La croissance démographique mondiale engendre, de plus en plus, une forte consommation d'énergie. Les énergies fossiles: les hydrocarbures et le charbon fournissent $86 \%$ de l'énergie utilisée. Les reste est fournit par le nucléaire et les énergies renouvelables.

Les énergies fossiles sont non renouvelables. En plus de ce principal caractère, Leur extraction et utilisation engendrent d'énormes problèmes à l'environnement et entraînent une augmentation des concentrations atmosphériques en Gaz à Effet de Serre (GES). Face à la nécessité de réduire ces émissions mondiales de GES, les pays sont amenés à revoir leurs politiques énergétiques en s'appuyant sur les énergies renouvelables. L'Algérie ne déroge pas à cette règle, elle amorce un ambitieux programme de développement des énergies renouvelables.

Ces énergie sont des énergies d'avenir inépuisables, fournies par le soleil, le vent, la chaleur de la Terre, l'eau ou encore les végétaux. Leur exploitation engendre peu d'émissions polluantes.

A cet effet, notre problématique est la suivante : quel avenir pour les énergies renouvelables en Algérie?

Pour répondre à cette question principale, nous allons essayer de répondre à ces trois questions sous-jacentes:

- quelle est la liste des énergies renouvelables qui s'offre à nous ?

- quel est le potentiel de ces énergies en Algérie ?

- quelle est la stratégie de l'Algérie dans ce domaine ?

Mots clés: énergies renouvelables, Algérie, potentiel énergétique, stratégie énergétique

\section{Mme IGUERGAZIZ Wassila}

Maitre de conférences à la faculté des sciences économiques de gestion et des sciences commerciales, Université Mouloud MAMMERI de Tizi-ouzou Algérie

Banque_dz@yahoo.fr

Tél : 00213553329398 


\section{Introduction}

Le mot « énergie » vient du grec ancien « energeia », qui signifie « force en action ». L'énergie est tout ce qui permet d'effectuer un travail, de produire de la lumière, de la chaleur ou un mouvement. A noter qu'elle peut être qualifiée de «renouvelable $»$ ou de « non renouvelable ${ }^{1}{ }^{1}$

On parle des énergies renouvelables par opposition aux énergies fossiles tels que le charbon, le pétrole, le gaz naturel... Ce sont des énergies inépuisables produites à partir de phénomènes naturels théoriquement illimitées, ou reconstituables plus rapidement qu'elles ne sont consommées. Leur exploitation n'engendre pas ou peu de déchets et d'émissions polluantes.

Ce sont les énergies de l'avenir, mais elles sont sous-exploitées par rapport à leur potentiel et ne couvrent que $20 \%$ de la consommation mondiale d'électricité. ${ }^{2}$

\section{Les différentes sources d'énergies renouvelables}

Ces énergies sont également désignées par les termes « énergies vertes 》 ou " énergies propres ». Elles sont fournies par le vent, l'eau, le soleil, la chaleur de la Terre, encore la croissance des végétaux.

\subsection{L'énergie éolienne}

Le vent est une puissante source d'énergie, et l'une des premières à avoir été utilisée par l'humanité. En effet, l'énergie éolienne ${ }^{3}$ est exploitée depuis des milliers d'années au travers des moulins à vent et de la navigation.

D'abord utilisée pour la navigation à voile dans l'Egypte ancienne, 3000 ans avant Jésus-Christ. Ensuite, elle donne naissance aux premiers moulins à vent dans les années 600, dans une région correspondant maintenant à l'Iran. Le moulin à vent

\footnotetext{
${ }^{1}$ Les énergies non renouvelables sont des énergies fossiles, constituées de substances qui mettent des millions d'années à se reconstituer, par exemple : charbon, gaz, pétrole. II existe une autre forme d'énergie non renouvelable : l'énergie nucléaire, produite à partir d'un minerai appelé uranium.

2 Énergies renouvelables: définition, exemples, avantages et limites, https://erse.net/definitions/energies-renouvelables-definition/\#gs.p4usk0

${ }^{3}$ Dans la mythologie grecque, Éole est le maître des vents. Du nom d'Éole dérivent l'adjectif « éolien » et le nom « éolienne ». https://fr.wikipedia.org/wiki/\%C3\%89ole
} 
qui sert à moudre le grain, va s'exporter en Europe, notamment en Grèce au $12^{\mathrm{ème}}$ siècle, puis en Chine au $13^{\mathrm{ème}}$ siècle. Dans les années 1700 , les turbines éoliennes apparaissent au Canada grâce aux colons qui les utilisent pour irriguer les terres. Avec la révolution industrielle du $19^{\text {ème }}$ siècle, de nouveaux concepts apparaissent et modernisent le moulin à vent. Le $20^{\mathrm{ème}}$ siècle, en particulier à partir de la crise pétrolière des années 1970, ravive l'intérêt pour les énergies renouvelables et marque le développement de l'industrie éolienne, qui représente à la fin du siècle un chiffre d'affaires d'environ 7 milliards de dollars. ${ }^{4}$

Aujourd'hui, nous pouvons exploiter cette énergie à l'aide de machines qui le transforment en énergie électrique. En effet, une éolienne est un dispositif mécanique qui convertit l'énergie cinétique ${ }^{5}$ du vent en électricité. Elle est composée d'un mât, une nacelle et un rotor.

Le mât, d'une centaine de mètres de hauteur, soutient la nacelle. Cette dernière capte des vents plus hauts donc plus forts. Elle abrite également la génératrice. Quant au rotor, c'est un élément auquel sont fixées les trois pales qui entrent en mouvement rotatif grâce à l'intensité du vent et fait ainsi tourner un arbre mécanique. Cette énergie est enfin convertie en électricité par la génératrice, elle est ensuite transportée par câble souterrain jusqu'au poste de livraison, afin d'être injecté sur le réseau national. ${ }^{6}$

Les éoliennes sont installées dans des endroits où le vent atteint une vitesse élevée. Elles produisent de l'électricité lorsque la vitesse du vent se situe entre 3 et 25 mètres par seconde. Lorsque le maximum de 25 mètres est atteint, le rotor est freiné par un dispositif, ce qui conduit à un arrêt de la machine tant que le vent ne faiblit pas.

En Australie, d'après une étude menée en 2018 par le professeur Bruce Mountain du Victoria Energy Policy Centre, les énergies solaire et principalement éolienne réduisent le prix de gros de l'électricité de 30\%.

\footnotetext{
${ }^{4}$ La petite histoire de l'énergie du vent.

5 "L'énergie cinétique est l'énergie que possède un corps du fait de son mouvement. L'énergie cinétique d'un corps est égale au travail nécessaire pour faire passer le dit corps du repos à son mouvement ». https://www.futura-sciences.com/sciences/definitions/physique-energie-cinetique-9430/ ${ }^{6}$ Planète healthy, énergie éolienne.
} 
Il existe plusieurs types d'éoliennes : les éoliennes terrestres, les éoliennes offshore $^{7}$, les éoliennes off-shore flottantes ${ }^{8}$ mais le principe reste globalement le même pour tous ces types.

Figure $\mathrm{n}^{\circ} 1$ : les différents types d'éoliennes

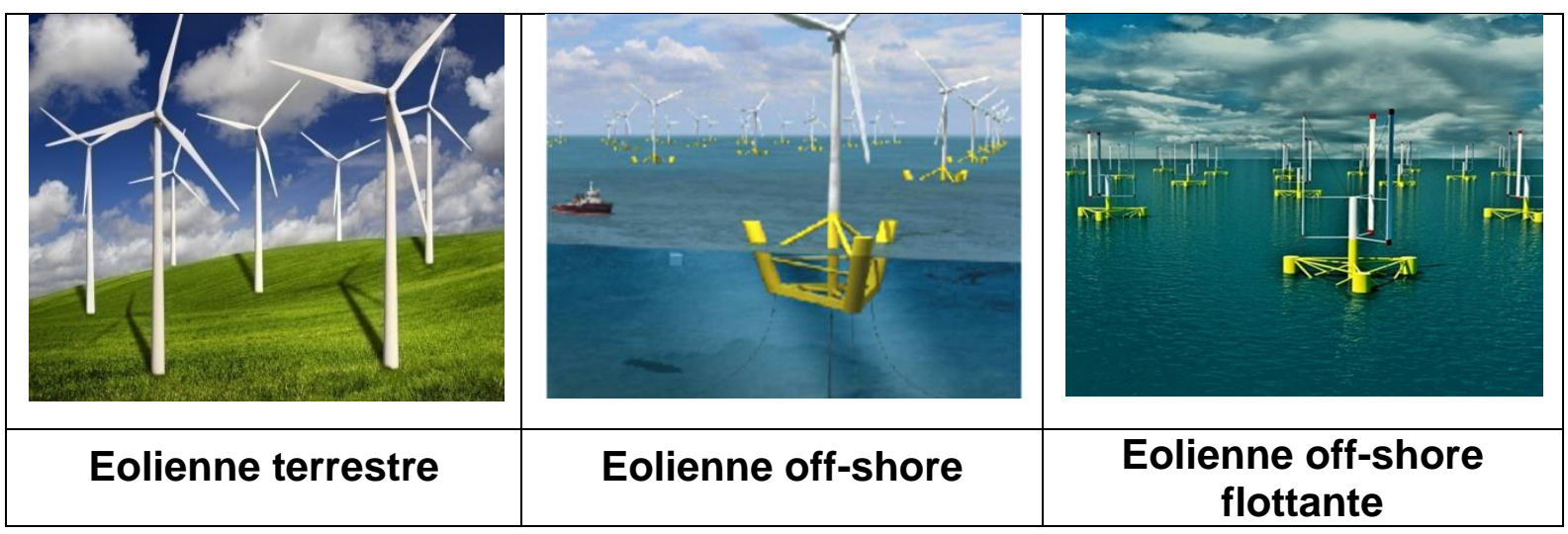

\subsection{L'énergie hydraulique}

L'énergie hydraulique est l'énergie fournie par le mouvement de l'eau, sous toutes ses formes : chute, cours d'eau, courant marin, marée, vagues.

Les premiers moulins à eau construits par les Grecs vers 300-250 AJC étaient de petite dimension et à usage domestique. Ce type de moulin fut utilisé à travers I'Europe jusqu'à la fin du Moyen Âge. À l'origine, ils servent essentiellement à moudre les grains, presser les olives, broyer les noix ou les raisins...etc. Par la suite, ils sont appliqués à toutes sortes d'industries et sont devenus une étape décisive pour l'utilisation artisanale et industrielle de l'énergie hydraulique. Ils symbolisent la toute première révolution industrielle qui commence au IXème siècle au moyen âge, et ce, en augmentant la productivité et en s'adaptant à toutes types d'industries.

\footnotetext{
7 Offshore" en anglais signifie "hors côtes", par opposition à "on-shore" qui caractérise une éolienne terrestre. Une éolienne offshore est donc une éolienne en mer. https://www.google.com/search?q=diff\%C3\%A9rence+entre+\%C3\%A9olienne+offshore+et+onshore\& oq=diff\%C3\%A9rence+entre+\%C3\%A9olienne+off-

shore+\&aqs=chrome.1.69i57j0|3.25596j0j1 \&sourceid=chrome\&ie=UTF-8

${ }^{8}$ L'éolienne offshore est ancrée à une fondation, son installation nécessite des travaux importants sur place, et des navires spécialisés pour l'opération. Dans le cas de l'éolienne offshore flottant, les fondations sous-marines sont remplacées par un flotteur qui peut être relié au fond par de simples lignes d'ancrage. De même, son montage peut être réalisé au port pour être remorqué ensuite sur le site. https://www.lenergietoutcompris.fr/actualites-et-informations/politique-energetique/l-eolien-flottant-vs-leolien-pose-quelles-differences-48193
} 
La production de l'électricité commence au cours du XIXème siècle. À la fin du siècle, la turbine ${ }^{9}$ remplacera la roue hydraulique et les premiers barrages feront leur apparition. Entre 1920 et 1940, plus de cinquante barrages sont édifiés. De nos jours, I'hydroélectricité est l'énergie renouvelable la plus exploitée dans le monde entier. ${ }^{10}$

Le principe du fonctionnement est simple, le poids et la vitesse de l'eau actionne une turbine qui entraîne à son tour un alternateur, l'énergie mécanique produite va être transformée par cet alternateur en énergie électrique. Enfin, l'électricité est dirigée vers le réseau électrique

Figure $\mathrm{n}^{\circ} 2$ : une centrale hydraulique

\section{Centrale hydraulique}

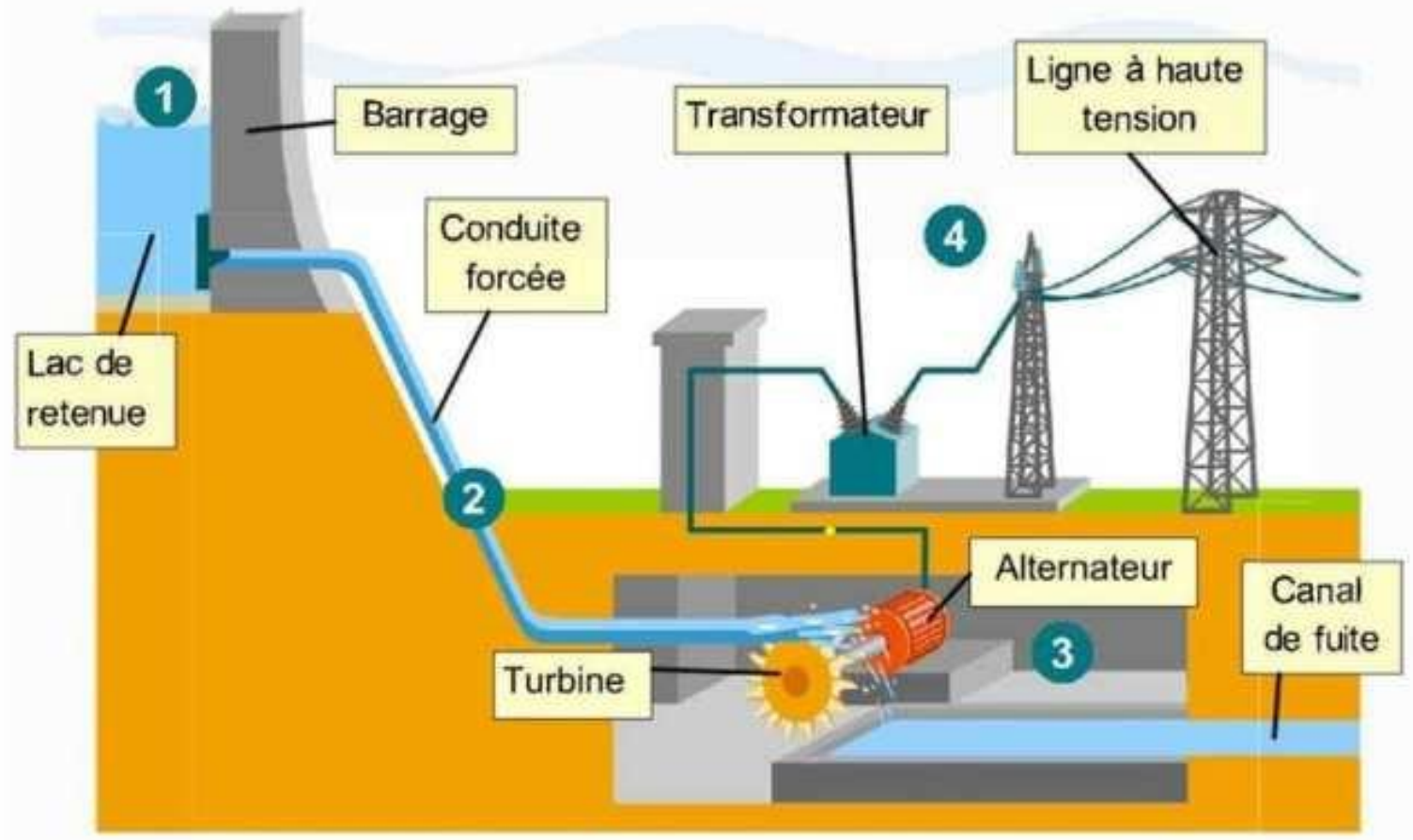

\subsection{L'énergie solaire}

\footnotetext{
${ }^{9}$ Benoist de Fourneyron (1802-1867) invente la première turbine. II utilise l'effet de la pression pour entraîner une roue à eau. II commence en 1827 avec une première turbine en Franche-Comté.

10 Les énergies renouvelables de la préhistoire à nos jours.
} 
Le soleil émet un rayonnement sous forme d'ondes électromagnétiques. En fonction de la longueur de ces ondes, certaines sont visibles (la lumière) et d'autres non (infrarouges, ultraviolets). ${ }^{11}$ Bien que les rayons du soleil soient observés depuis la nuit des temps, leur composition ne commence à être étudiée qu'à partir du XVIIème siècle. En effet, dans les années 1660, Isaac Newton décompose le rayonnement solaire, il estime alors qu'il est composé de particules (théorie corpusculaire), contrairement à son contemporain Christiaan Huygens, qui le visualise sous forme d'ondes (théorie ondulatoire). Au XIXème siècle, la théorie ondulatoire de la lumière s'impose aux dépens de la théorie corpusculaire.

Cependant, au début du XXème siècle, Albert Einstein découvre que les rayons se diffusent bien sous forme d'ondes, mais ils sont aussi composés de photons. On parle désormais de "dualité onde-corpuscule ». ${ }^{12}$

Le soleil est une source quasiment inépuisable d'énergie qui envoie à la surface de la terre un rayonnement qui représente chaque année environ 8400 fois la consommation énergétique de l'humanité. Les déserts de notre planète reçoivent en 6 heures plus d'énergie du soleil que n'en consomme l'humanité en une année. ${ }^{13}$

Les rayonnements solaires sont capables d'être transformés en deux formes d'énergie : énergie thermique (chaleur) et énergie photovoltaïque (électricité) ${ }^{14}$.

\subsubsection{L'énergie thermique}

Le mot thermique vient du grec thermos, qui signifie « chaleur ». Le soleil à toujours servi à l'homme pour se chauffer, sans utiliser aucune invention.

Les panneaux solaires thermiques permettent de produire de l'eau chaude. Un panneau solaire thermique est une surface qui capte les rayons du soleil pour réchauffer un fluide caloporteur placé sous les panneaux. Ce fluide chaud rejoint ensuite le ballon de stockage dans lequel il réchauffe l'eau chaude sanitaire

\section{Figure 3 : Solaire thermique}

\footnotetext{
${ }^{11}$ Les infrarouges et les ultraviolets sont découverts respectivement par l'Allemand William Herschel (1738-1822) en 1800, et par son compatriote Johann Wilhelm Ritter (1776-1810) peu de temps après.

12 Einstein a reçu le prix Nobel de Physique en 1921 pour cette découverte.

$13 \mathrm{https}: / /$ rs.umc.edu.dz/umc/Production_Photoviltaique_BF.pdf

${ }^{14}$ Les énergies renouvelables de la préhistoire à nos jours.
} 


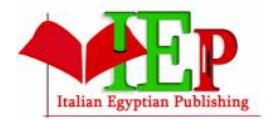

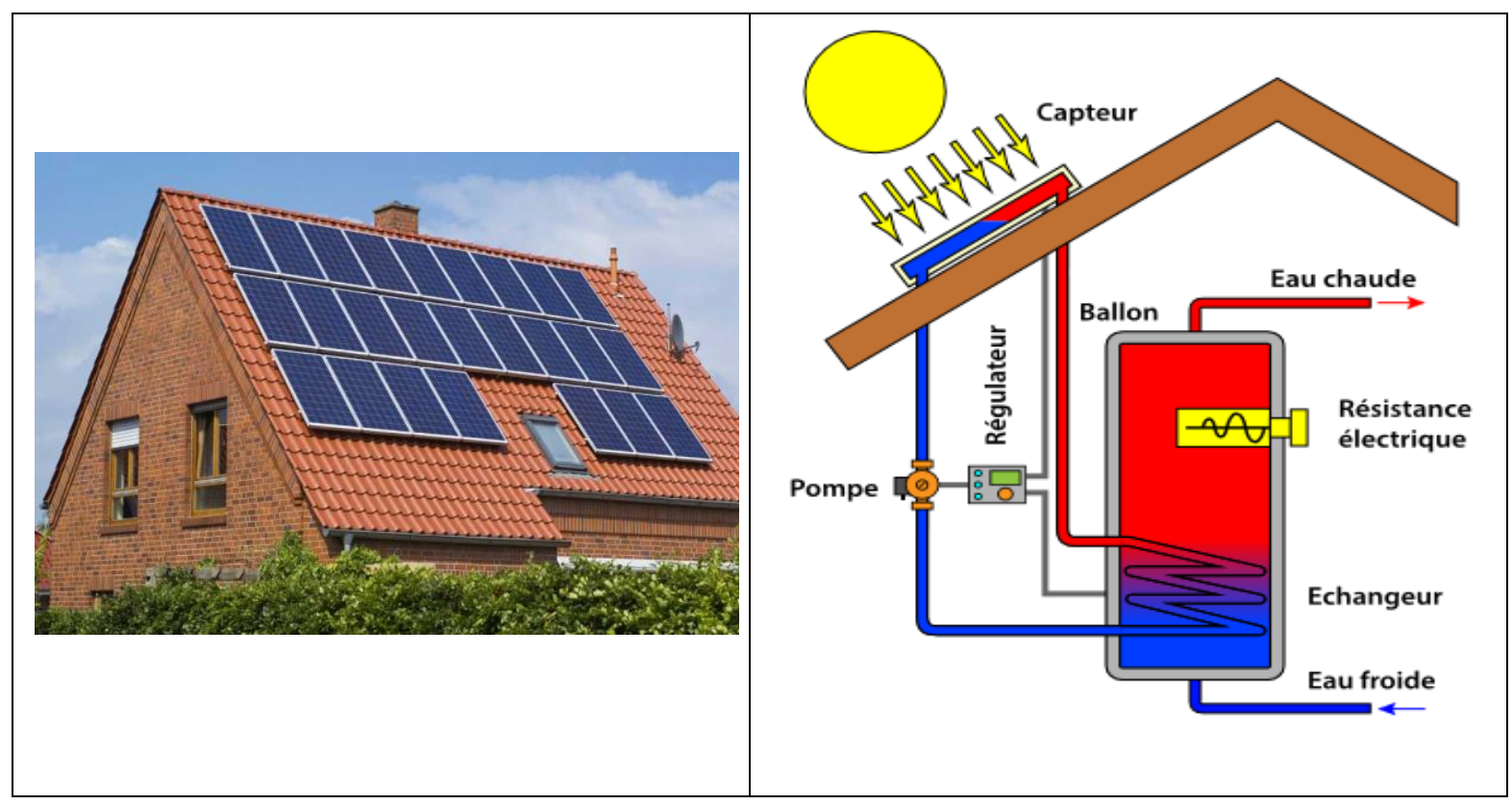

\subsubsection{L'énergie photovoltaïque}

Le solaire photovoltaïque est une source d'électricité nécessitant un moyen de stockage ou une liaison avec le réseau électrique. Ce type d'énergie renouvelable est issu directement de la captation du rayonnement solaire.

Le photovoltaïque a l'avantage de produire de l'énergie là où elle est consommée. On peut implanter des panneaux photovoltaïques sur des surfaces bâties exposées au soleil. ${ }^{15}$

Le principe d'une cellule photovoltaïque est de transformer des photons absorbés par un semi-conducteur en porteurs de charges électriques (électrons et trous). Cette création de charges va entraîner la création d'une différence de potentiel aux bornes d'électrodes et d'un courant électrique dans un circuit connecté aux électrodes.

Figure 4 : principe de fonctionnement d'une cellule photovoltaïque

15 http://www.enr.fr/userfiles/files/Brochures\%20G\%C3\%A9n\%C3\%A9rales/ENR\%20QuestionsR\%C3\%A9ponses\%20OK.pdf 


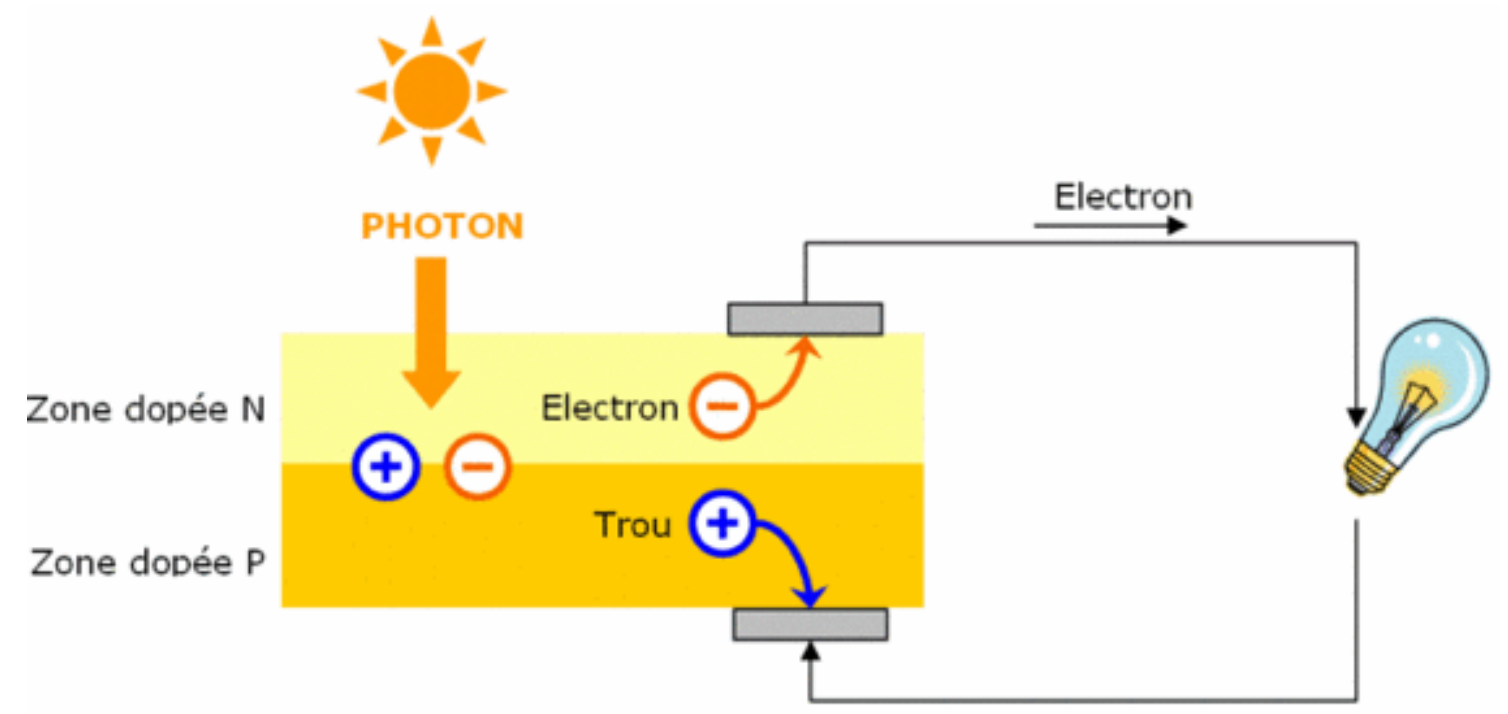

\subsection{La géothermie}

Etymologie de mot géothermie est grecque : "Gê" signifie la terre, et "Thermie", la chaleur. La géothermie consiste à capter la chaleur de la croûte terrestre pour produire du chauffage ou de l'électricité en faisant des forages, plus ou moins profonds selon la température désirée. Plus l'on fore profond plus la température augmente. En moyenne l'augmentation de température atteint 20 à 30 degrés par kilomètre.

Généralement on distingue trois types de géothermie selon le niveau de température disponible : la géothermie à haute énergie, la géothermie de basse énergie et la géothermie de très basse énergie

La géothermie à haute énergie : exploite des sources hydrothermales très chaudes, ou des forages très profonds. Cette géothermie est surtout utilisée pour produire de l'électricité. Elle est parfois subdivisée en deux sous-catégories : la géothermie moyenne énergie (températures comprises entre 100 et $150^{\circ} \mathrm{C}$ ) et la géothermie haute énergie (températures supérieures à $150^{\circ} \mathrm{C}$ ).

La géothermie de basse énergie : géothermie des nappes profondes aux températures situées entre 30 et $100^{\circ} \mathrm{C}$. La principale utilisation est les réseaux de chauffage urbain.

La géothermie de très basse énergie : géothermie des faibles profondeurs aux niveaux de température compris entre 10 et $30^{\circ} \mathrm{C}$. Principales utilisations : le chauffage et la climatisation individuelle 
Figure 5 : la géothermie

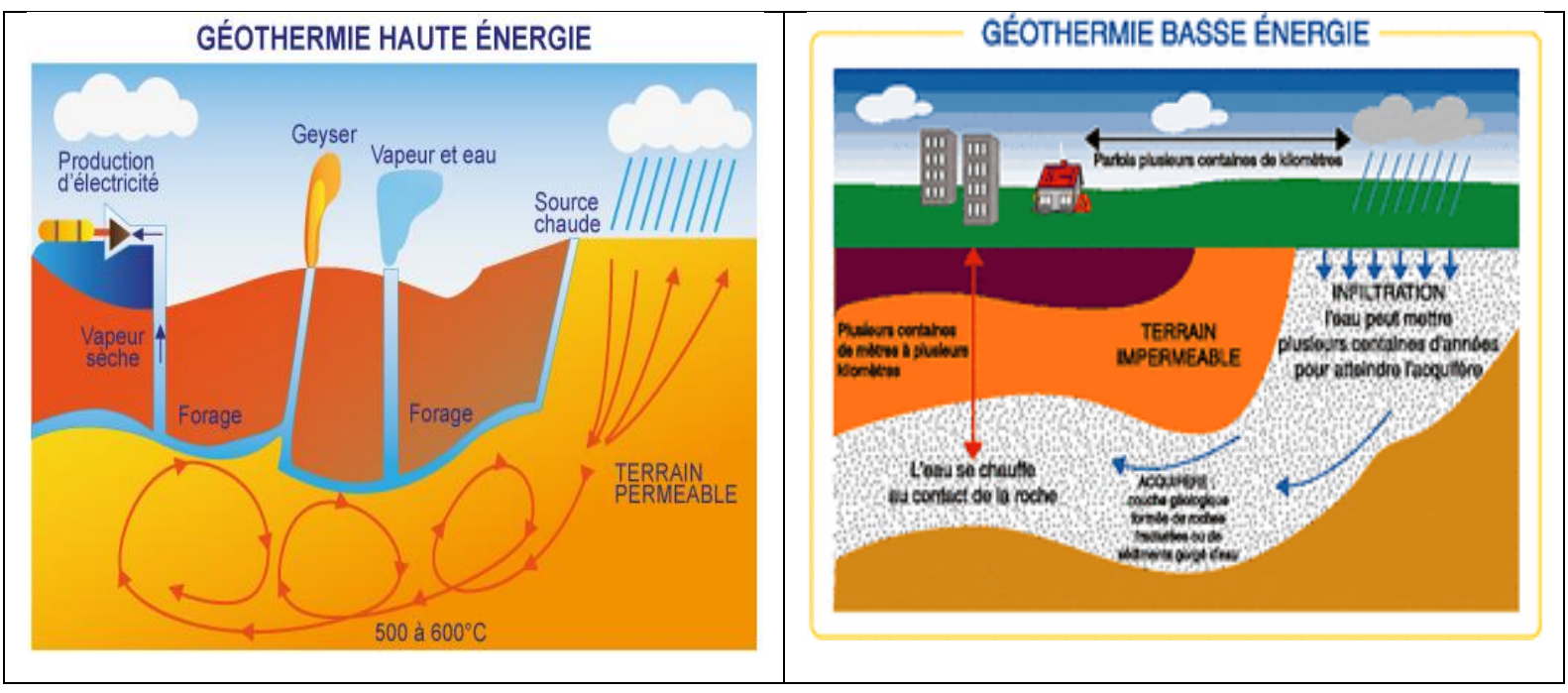

\subsection{La biomasse}

Les matières organiques végétales ou animales peuvent aussi être utilisées pour produire de l'énergie. On appelle cette source d'énergie la biomasse.

La biomasse signifie la matière issue du vivant (Bio = vivant, Masse matière) C'est la première source d'énergie exploitée pendant des millénaires comme source d'énergie lumineuse et thermique. Son exploitation remonte à la préhistoire par I'utilisation du bois pour se chauffer, s'éclairer et cuire les aliments. ${ }^{16}$

On en distingue trois catégories principales : le bois, le biogaz, les biocarburants. Le bois énergie représente $14 \%$ de la consommation énergétique mondiale. Il est brûlé pour produire de la chaleur. Le biogaz est issu de la fermentation des déchets organiques. Sa combustion produit de la chaleur, mais également de l'électricité par cogénération. Quant aux biocarburants, ils proviennent de plantes cultivées (tournesol, betterave, colza...). Le biodiesel, l'éthanol, et son dérivé, sont les plus courants. Ils sont mélangés à de l'essence ou à du gazole. ${ }^{17}$

\section{Le potentiel des énergies renouvelables en Algérie}

\footnotetext{
$16 \mathrm{http}: / /$ www.master-prnt.com/qse_dd/pdf/2007/rapport_energiesrenouvelables.pdf

$17 \mathrm{http}: / /$ www.energies-renouvelables.org/media/photos/observ-er/5familles/5_familles_ER.pdf
} 
L'Algérie s'engage dans une nouvelle ère énergétique durable afin d'apporter des solutions aux défis environnementaux, à travers le lancement d'un programme pour le développement des énergies renouvelables. Ce denier a été adopté par le gouvernement en février 2011 et révisée en mai 2015. II est placé au rang de priorité nationale en février 2016 lors du conseil du restreint gouvernement. ${ }^{18}$

Le programme des énergies renouvelables consiste à installer une puissance d'origine renouvelable de l'ordre de $22.000 \mathrm{MW}$ à l'horizon 2030 pour le marché national, avec l'option stratégique de l'exportation, si les conditions du marché le permettent. Ce programme permettra de réduire les émissions de CO2 de 193 millions de tonnes. ${ }^{19}$

C'est dans cette optique que l'Algérie ainsi que neuf pays arabes ${ }^{20}$ ont créé, en vertu de la déclaration du Caire, signée en juin 2008, le Centre Régional pour les Énergies Renouvelables et l'Efficacité Énergétique en anglais (RCREEE)

Cette organisation régionale indépendante vise à faciliter et promouvoir l'adoption des pratiques d'énergie renouvelable et d'efficacité énergétique dans le monde arabe. Le RCREEE a acquis son statut juridique d'une organisation internationale indépendante à but non lucratif, en août 2010, à travers la conclusion d'un accord de siège avec le gouvernement égyptien. ${ }^{21}$

\subsection{Le potentiel de l'énergie solaire en Algérie}

L'Algérie a un grand potentiel en énergies renouvelables, elle dispose d'un des gisements solaire les plus élevés au monde. La durée d'insolation dépasse les 2000 heures annuellement et peut atteindre les 3900 heures au Sahara et aux hauts plateaux.

\section{Carte 1 : le potentiel de l'énergie solaire}

\footnotetext{
${ }^{18} \mathrm{https}: / /$ www.energy.gov.dz/?rubrique=energies-nouvelles-renouvelables-et-maitrise-de-Irenergie 19 Idem

20 L'Egypte, le Maroc, la Tunisie, la Lybie, le Liban, le Yémen, la Jordanie, Palestine, la Syrie

${ }^{21} \mathrm{http}: / /$ www.energy.gov.dz/francais/uploads/2016/Energie/energie-renouvelable.pdf
} 


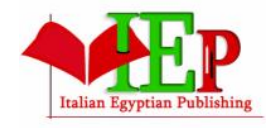

ISSN: 2735-5403
INTERNATIONAL JOURNAL OF

ADVANCED RESEARCH ON PLANNING AND

SUSTAINABLE DEVELOPMENT

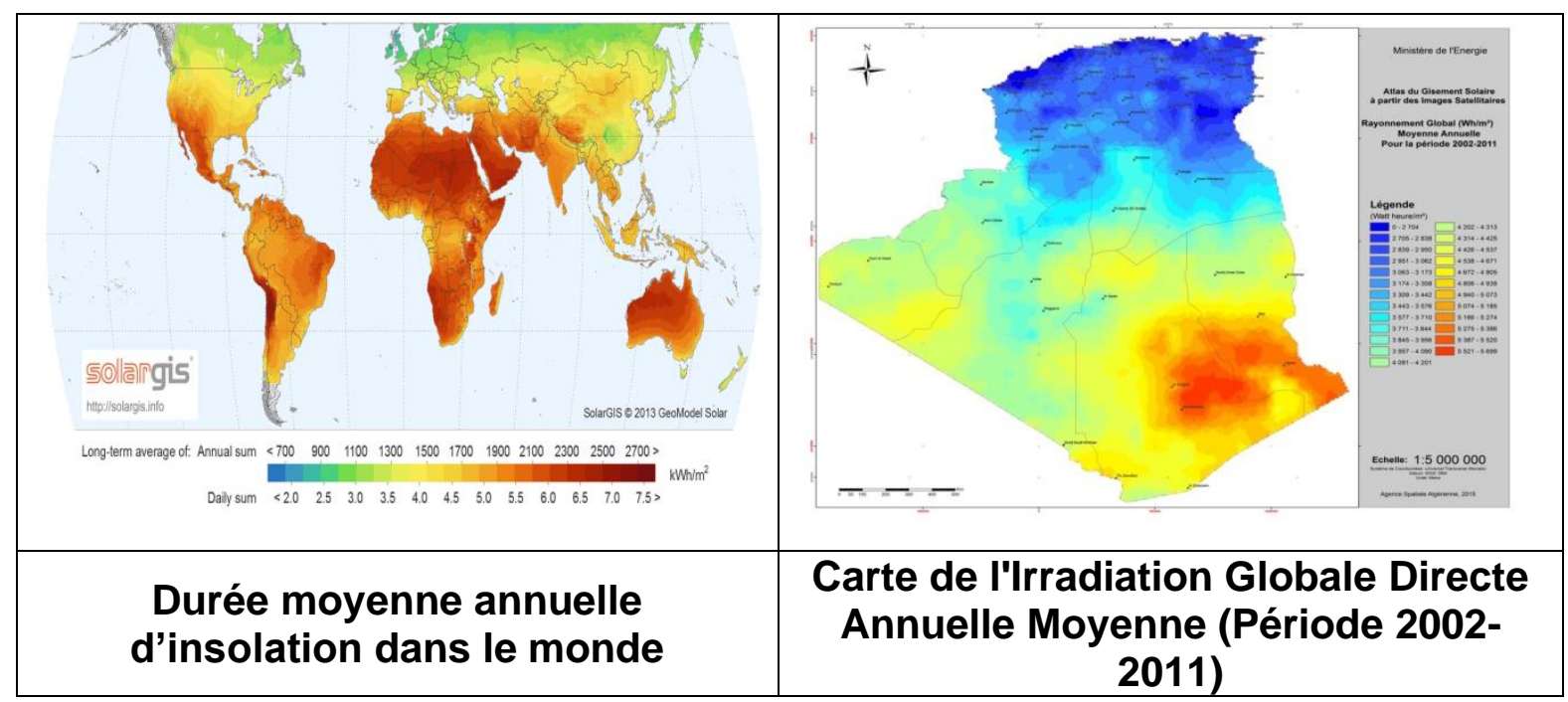

\subsection{Le potentiel de l'énergie éolienne en Algérie}

L'Algérie jouit de ressources éoliennes abondantes, elle dispose de nombreuses zones qui bénéficient de vents forts de $7-9 \mathrm{~m} / \mathrm{s} .{ }^{22}$ cette ressource varie beaucoup d'un endroit à un autre.

En effet, l'Algérie se subdivise en deux grandes zones géographiques distinctes. Le Nord méditerranéen avec un littoral de $1200 \mathrm{Km}$ et les deux chaines montagneuses: l'Atlas tellien et l'Atlas saharien. Entre celles-ci s'intercalent des plaines et les hauts plateaux de climat continental. Au nord, globalement la vitesse moyenne est peu élevée. Sur les hauts plateaux de Tébessa, Biskra, M'sila et El bayadh la vitesse varie entre 6 à $7 \mathrm{~m} / \mathrm{s}$.

Le Sud, quant à lui, se caractérise par un climat saharien et par des vitesses plus élevées que le Nord, plus particulièrement dans le Sud-est, avec des vitesses supérieures à $7 \mathrm{~m} / \mathrm{s}$ et qui dépassent la valeur de $8 \mathrm{~m} / \mathrm{s}$ dans la région de Tamanrasset (In Amguel).

\section{Carte 2 : vitesse de vent moyenne}

\footnotetext{
${ }^{22}$ Une éolienne produit de l'électricité lorsque la vitesse du vent se situe entre 3 et 25 mètres par seconde.
} 


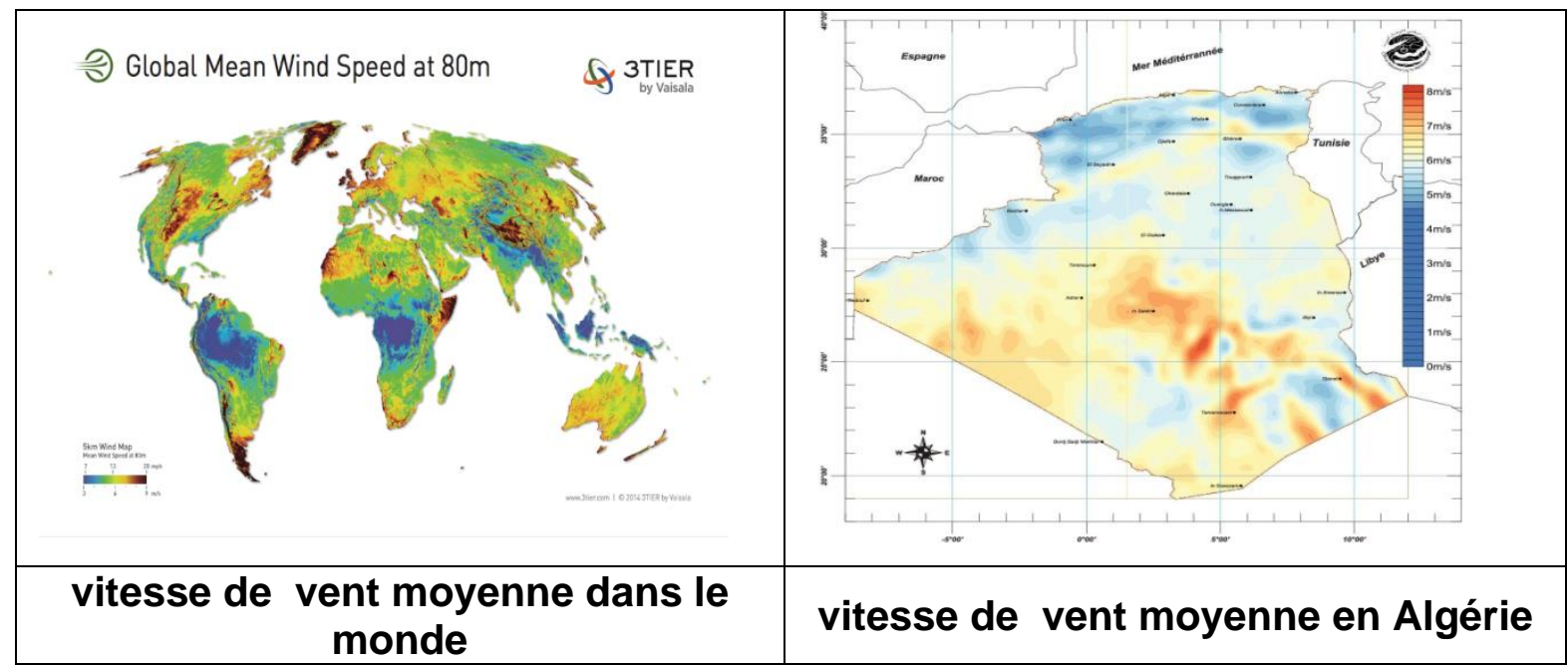

\subsection{Le potentiel de l'énergie géothermique en Algérie}

Les données géologiques ont permis d'identifier plus de 200 sources chaudes qui ont été inventoriées dans la partie nord du pays. Un tiers environ (33\%) d'entre elles ont des températures supérieures à $45^{\circ} \mathrm{C}$, dont certaines peuvent atteindre $118^{\circ} \mathrm{C}$ à Biskra.

Des études ont également permis d'identifier trois zones dont le gradient ${ }^{23}$ dépasse les $5^{\circ} \mathrm{C} / 100 \mathrm{~m}$ :

- Zone de Relizane et Mascara;

- Zone d'Aïne Boucif et Sidi Aïssa ;

- Zone de Guelma et Djebel El Onk. ${ }^{24}$

\subsection{Le potentiel de l'énergie hydraulique en Algérie}

L'importante quantité tombant sur le territoire algérien est estimée à 65 milliards de $\mathrm{m}^{3}$, mais elle profite peu au pays en raison de nombre réduit de jours de précipitation, concentration sur des régions limitées (les ressources décroissent du nord au sud), forte évaporation, évacuation rapide vers la mer.

103 sites de barrages ont été recensés. Plus de 50 barrages sont actuellement en exploitation. ${ }^{25}$

\footnotetext{
${ }^{23}$ Le gradient est l'augmentation de la température des roches avec la profondeur, est la valeur normale est de l'ordre de $3{ }^{\circ} \mathrm{C} / 100 \mathrm{~m}$

${ }^{24} \mathrm{https}: / / w w w . e n e r g y . g o v . d z /$ ?rubrique=energies-nouvelles-renouvelables-et-maitrise-de-Irenergie

${ }^{25} \mathrm{https}: / / w w w . e n e r g y . g o v . d z /$ ?rubrique=energies-nouvelles-renouvelables-et-maitrise-de-Irenergie
} 


\subsection{Le Potentiel de la Biomasse}

Le potentiel de la forêt actuel est évalué à environ 37 millions tonnes équivalent pétrole (TPE). Le potentiel récupérable est de l'ordre de 3,7 millions de TEP et le taux de récupération actuel est de l'ordre de $10 \%{ }^{26}$

Le potentiel national des déchets ménagers est estimé à 05 millions de tonnes de déchets urbains et agricoles qui ne sont pas recyclés. Ce potentiel représente un gisement de l'ordre de 1.33 millions de TEP/an. ${ }^{27}$

\section{La stratégie De l'Algérie en matière des énergies renouvelables}

La stratégie de l'Algérie vise à développer une véritable industrie des énergies renouvelables. A travers ce programme, l'Algérie compte produire de l'électricité à partir des filières photovoltaïque, éolienne, la biomasse, la cogénération, la géothermie et le solaire thermique à partir de 2021. Ces filières seront les moteurs d'un développement économique durable, elle procure $27 \%$ de la production d'électricité destinée à la consommation nationale, d'ici 2030.

Le potentiel national en énergies renouvelables étant fortement dominé par le solaire, cela n'exclut pas pour autant le lancement de nombreux projets de réalisation de fermes éoliennes et la mise en œuvre de projets expérimentaux en biomasse, en géothermie et en cogénération.

\subsection{Le programme de développement des énergies renouvelables}

La réalisation des projets de production de l'électricité dédiés au marché national, dans le cadre du programme de développement des énergies renouvelables sera menée en deux étapes:

2015 - 2020 : une puissance de 4010 MW sera réalisée entre photovoltaïque et éolien, ainsi que $515 \mathrm{MW}$, entre biomasse, cogénération et géothermie.

\footnotetext{
${ }^{26}$ Bilan 2010, Direction Générale des forets.

${ }_{27}$ Caractérisation des déchets ménagers et assimilés dans les zones nord, semi-aride et aride d'Algérie 2014, Agence Nationale des Déchets
} 
2021 - 2030 : l'installation de grandes centrales d'énergies renouvelables dans les régions d'In Salah, Adrar, Timimoune et Bechar et leur intégration dans le système énergétique national afin de développer l'interconnexion électrique entre le Nord et le Sahara.

La réalisation pour le marché national sur la période 2015-2030 est de 22000 MW, répartie par filière comme suit:

Tableau 1 : la répartition par filière ${ }^{28}$

\begin{tabular}{|l|c|c|c|}
\hline \multicolumn{1}{|c|}{ Unité MW } & $\begin{array}{c}\mathbf{1} \text { ère } \text { phase } \\
\mathbf{2 0 1 5 - 2 0 2 0}\end{array}$ & $\begin{array}{c}\mathbf{2}^{\text {ème }} \mathbf{\text { phase }} \\
\mathbf{2 0 2 1 - 2 0 3 0}\end{array}$ & Total \\
\hline Photovoltaïque & 3.000 & 10.575 & 13.575 \\
\hline Eolien & 1.010 & 4.000 & 5.010 \\
\hline CSP & - & 2.000 & 2.000 \\
\hline Cogénération & 150 & 250 & 400 \\
\hline Biomasse & 360 & 640 & 1000 \\
\hline Géothermie & 05 & 10 & 15 \\
\hline Total & $\mathbf{4 . 5 2 5}$ & $\mathbf{1 7 . 4 7 5}$ & $\mathbf{2 2 . 0 0 0}$ \\
\hline
\end{tabular}

Figure 6 : réalisation par filière ${ }^{29}$

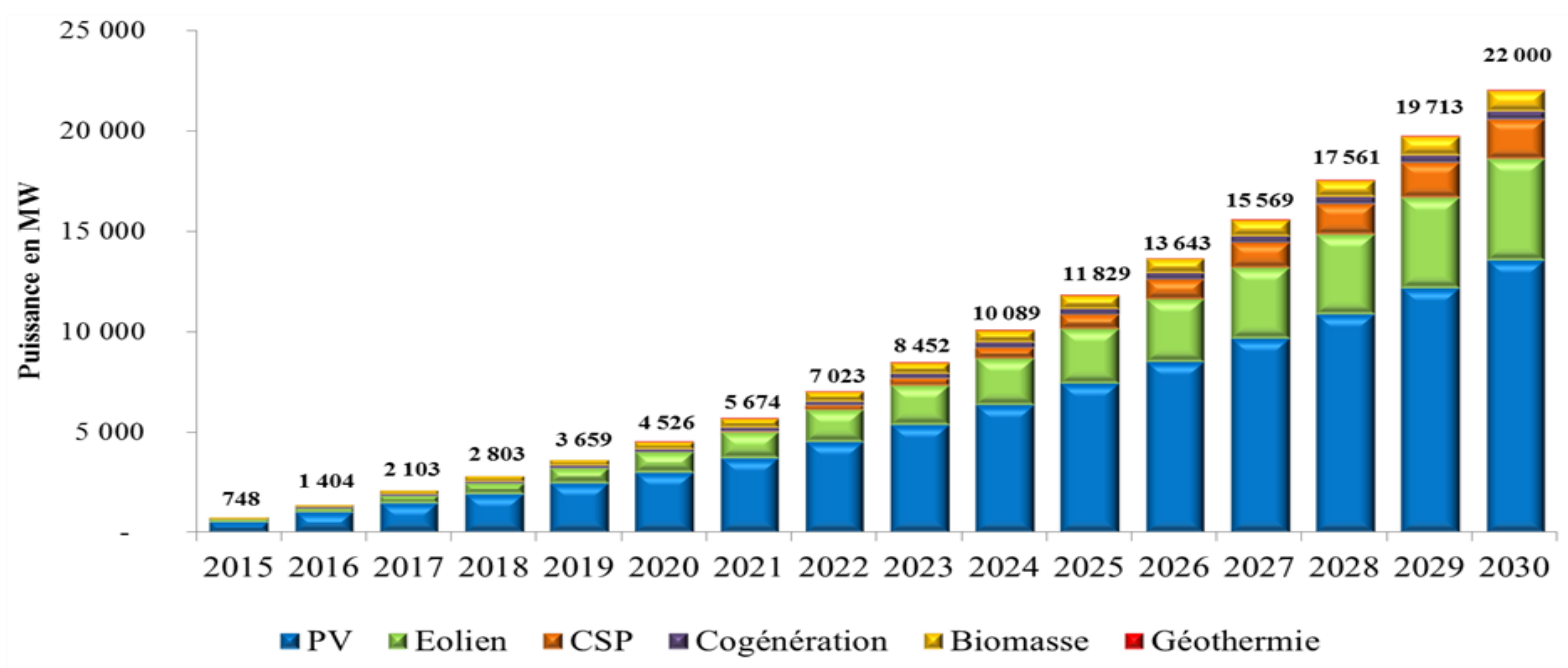

\subsection{Bilan des réalisations}

\footnotetext{
28 https://www.energy.gov.dz/?rubrique=energies-nouvelles-renouvelables-et-maitrise-de-Irenergie 29 Idem.
} 
Le programme a connu une phase d'expérimentation (2011-2014) dans laquelle plusieurs projets ont été réalisés

\section{Image 1 : Centrale Hybride Solaire-Gaz ${ }^{30}$}

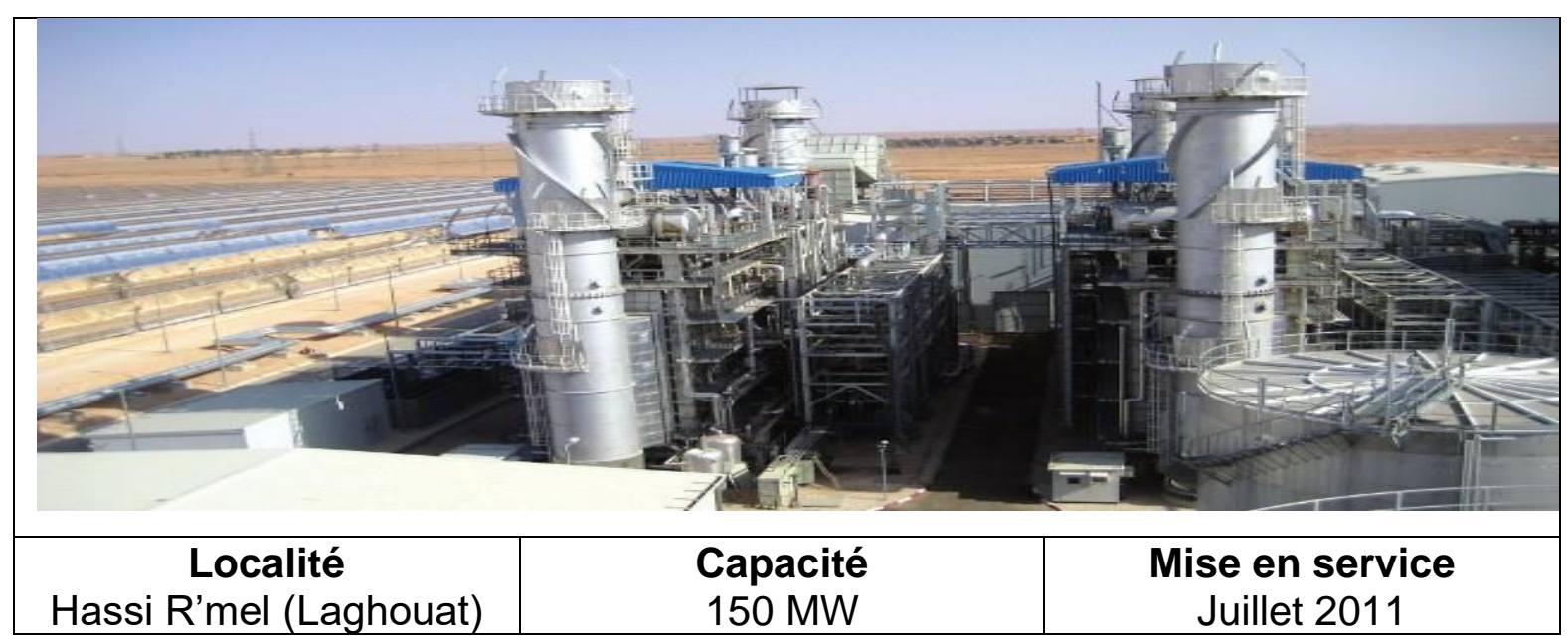

Image 2 : Ferme Eolienne ${ }^{31}$

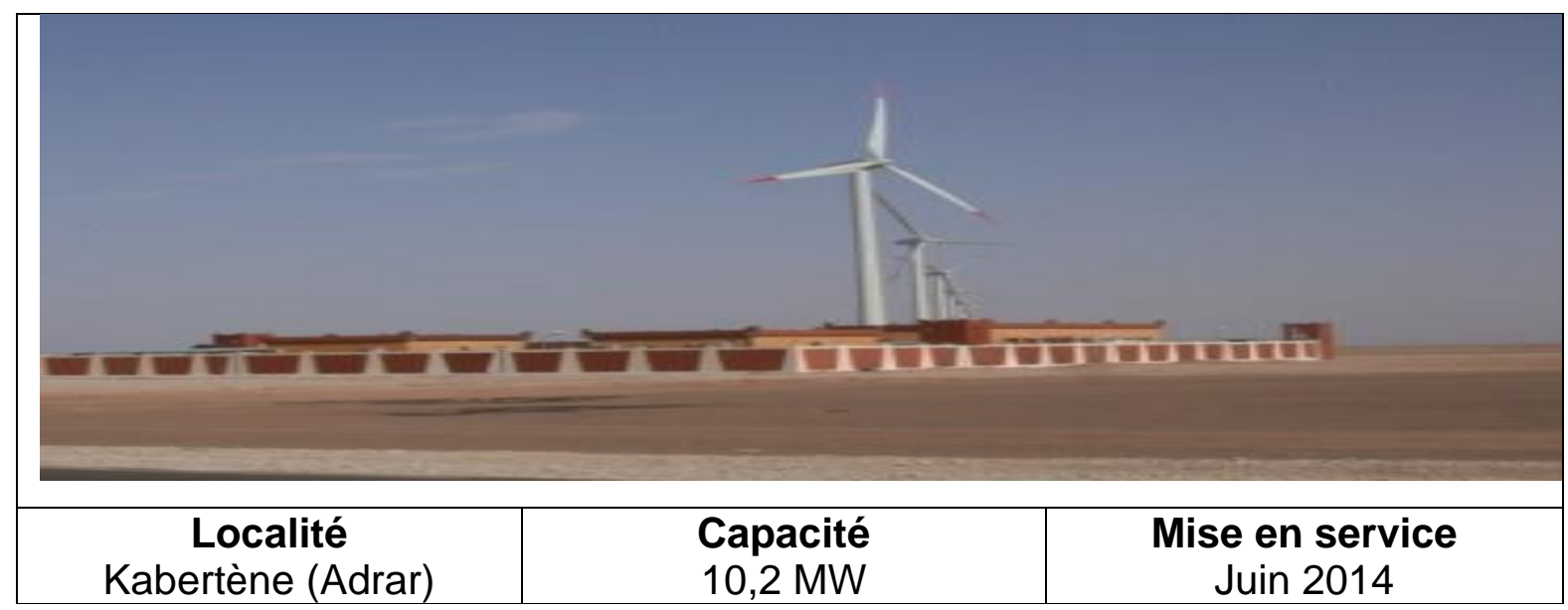

Image 3 : Centrale Pilote Photovoltaïque ${ }^{32}$

\footnotetext{
${ }^{30} \mathrm{https}: / / w w w . e n e r g y . g o v . d z /$ ?rubrique=energies-nouvelles-renouvelables-et-maitrise-de-Irenergie ${ }^{31}$ Idem.

32 Idem.
} 


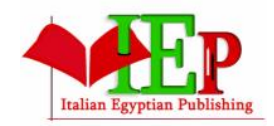

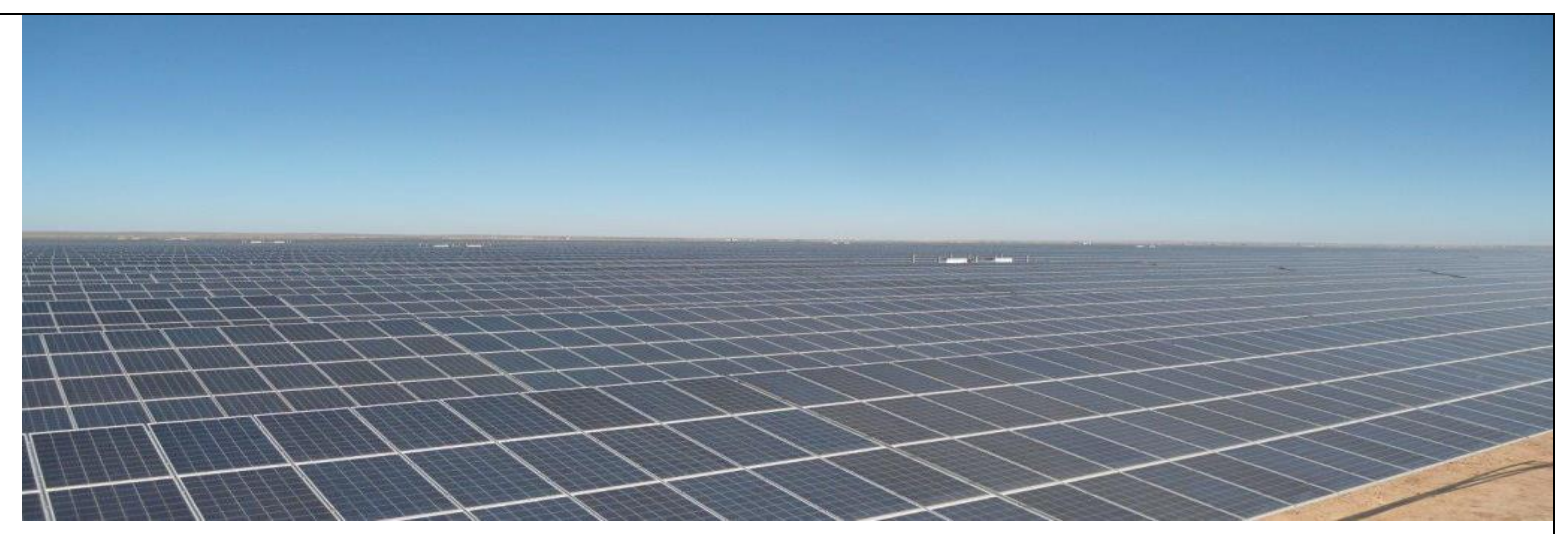

\begin{tabular}{c|c|c|}
\hline Localité & Capacité & Mise en service \\
juin 2014
\end{tabular}

l'électricité dédiés au marché national, dans le cadre du programme de développement des énergies renouvelables sera menée en deux étapes:

2015 - 2020 et 2021-2030. Ces deux étapes ont été précédées par une phase d'expérimentations, dont les projets réalisés sont présentés ci-dessus.

Quant aux réalisations relatives à la première phase, elles sont présentées dans le tableau en infra et illustrées par la carte ci-après :

Image 4 : Projet de 343 MWc en centrales photovoltaïques ${ }^{33}$

\footnotetext{
${ }^{33} \mathrm{https}: / / w w w . e n e r g y . g o v . d z /$ ?rubrique=energies-nouvelles-renouvelables-et-maitrise-de-Irenergie
} 


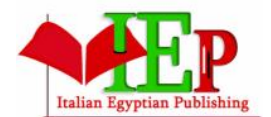

ISSN: $2735-5403$
INTERNATIONAL JOURNAL OF

ADVANCED RESEARCH ON PLANNING AND

\section{SUSTAINABLE DEVELOPMENT}

VOLUME 4, ISSUE 1, 2021, 46-68.

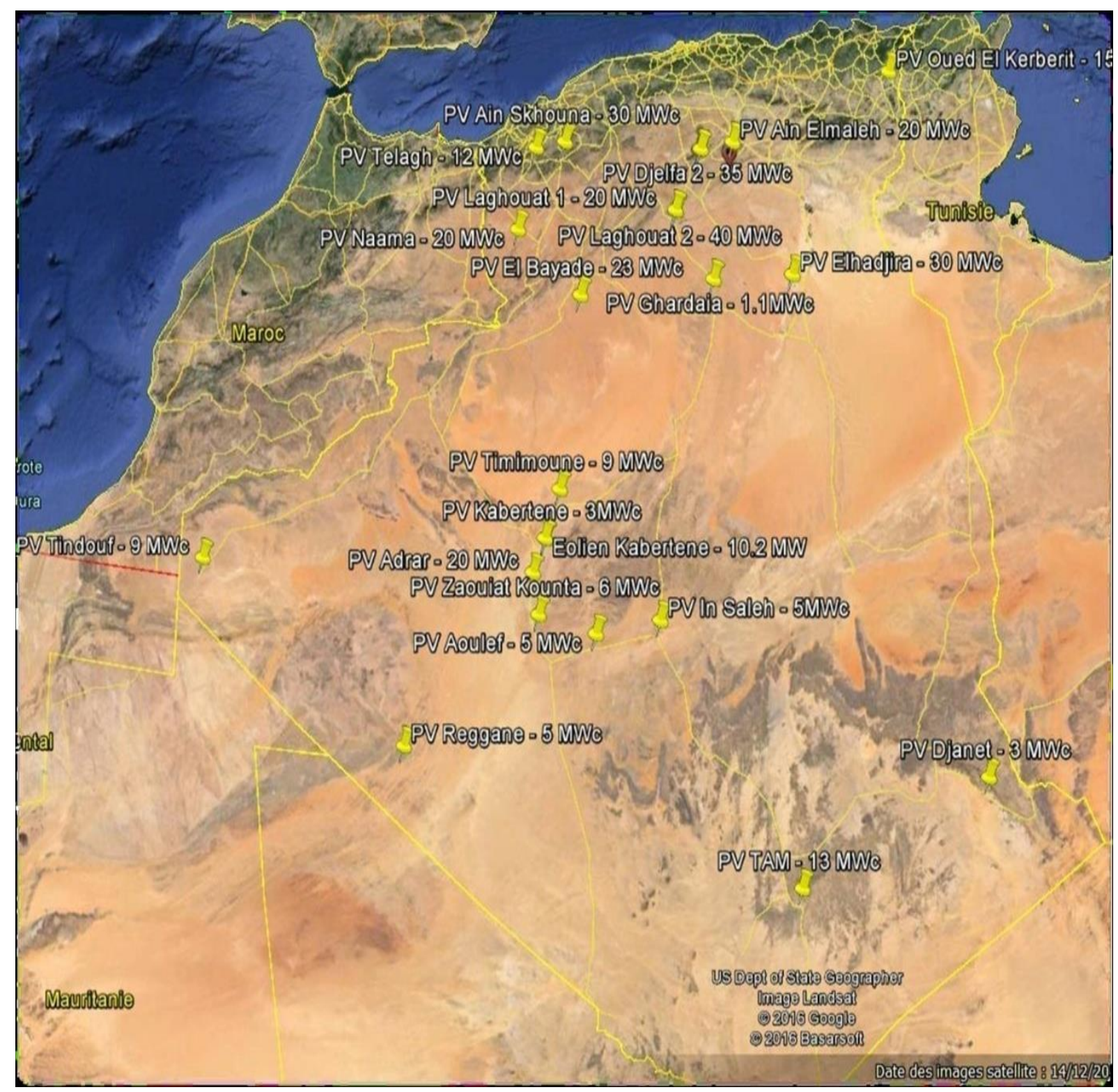

Tableau 2 : les réalisations de la première phase des centrales photovoltaïques 34

\begin{tabular}{|l|l|c|c|}
\hline \multicolumn{1}{|c|}{ wilaya } & \multicolumn{1}{|c|}{ Localité } & $\begin{array}{c}\text { Capacité } \\
\text { installée } \\
\text { MW }\end{array}$ & Mise en service \\
\hline Illizi & Djanet & 03 & $19 / 02 / 2015$ \\
\hline Adrar & Adrar & 20 & $28 / 10 / 2015$ \\
\hline
\end{tabular}

${ }^{34}$ https://www.energy.gov.dz/?rubrique=energies-nouvelles-renouvelables-et-maitrise-de-Irenergie 
INTERNATIONAL JOURNAL OF

ADVANCED RESEARCH ON PLANNING AND

SUSTAINABLE DEVELOPMENT

\begin{tabular}{|l|l|l|l|}
\hline Adrar & Kabertene & 03 & $13 / 10 / 2015$ \\
\hline Tamanrasset & Tamanrasset & 13 & $03 / 11 / 2015$ \\
\hline Tindouf & Tindouf & 09 & $14 / 12 / 2015$ \\
\hline Adrar & Zaouiet.Kounta & 06 & $11 / 01 / 2016$ \\
\hline Adrar & Reggane & 05 & $28 / 01 / 2016$ \\
\hline Adrar & Timimoune & 09 & $07 / 02 / 2016$ \\
\hline Tamanrasset & In-Salah & 05 & $11 / 02 / 2016$ \\
\hline Adrar & Aoulef & 05 & $07 / 03 / 2016$ \\
\hline Laghouat & El Khnag (I) & 20 & $08 / 04 / 2016$ \\
\hline Laghouat & El Khnag (II) & 40 & $26 / 04 / 2017$ \\
\hline Djelfa & Ain-El-Ibel (I) & 20 & $08 / 04 / 2016$ \\
\hline Djelfa & Ain-El-Ibel (II) & 33 & $06 / 04 / 2017$ \\
\hline Souk Ahras & Oued El Keberit & 15 & $24 / 04 / 2016$ \\
\hline Naâma & Sedrate Leghzal & 20 & $03 / 05 / 2016$ \\
\hline Saida & Ain-Skhouna & 30 & $05 / 05 / 2016$ \\
\hline Sidi-Bel-Abbes & Telagh & 12 & $29 / 09 / 2016$ \\
\hline Al Bayadh & Biodh Sidi Chikh & 23 & $26 / 10 / 2016$ \\
\hline M'sila & Ain-El-Melh & 20 & $26 / 01 / 2017$ \\
\hline Ouargla & El-Hdjira & 30 & $16 / 02 / 2017$ \\
\hline Batna & Oued El-Ma & 02 & $16 / 01 / 2018$ \\
\hline & & &
\end{tabular}

\subsection{Les résultats attendus}

Les résultats attendus par la réalisation de ce programme se présentent comme suit $: 35$

- un gisement d'économie d'énergie de l'ordre de 63 millions de TEP, soit près de 38 milliards de dollars ;

- une puissance évitée de plus de $1500 \mathrm{MW}$, soit près de 2 milliards de dollar ;

- une réduction de plus de 193 millions de tonnes de CO2, soit 1,1 milliards de dollar,

${ }^{35}$ Idem. 
- une création de 500.000 nouveaux postes d'emploi.

Tableau 3 : Tableau récapitulatif des émissions de $\mathrm{CO} 2$ à éviter à l'horizon $2030^{36}$

\begin{tabular}{|l|c|c|c|c|}
\hline $\begin{array}{l}\text { En millions } \\
\text { de tonnes } \\
\text { CO2 }\end{array}$ & 2015 & 2020 & 2025 & 2030 \\
\hline $\begin{array}{l}\text { Emissions } \\
\text { évitées }\end{array}$ & 1,1 & 32,1 & 95,9 & 193,3 \\
\hline
\end{tabular}

\subsection{Nouvelle stratégie pour le développement des énergies renouvelables pour} la production de l'électricité raccordée au réseau

En 2016, une nouvelle stratégie a été mise en place par le ministère de l'énergie pour le développement des énergies renouvelables. La mise en œuvre est régie par le décret exécutif n¹7-98 du 26 février 2017.

Cette stratégie est basée sur la procédure d'appels d'offres, afin d'accélérer la mise en œuvre du programme, vu la baisse des coûts des équipements sur le marché international. Elle permettra également de mettre en compétition les investisseurs dans le but de réduire le plus possible le prix du kWh produit à partir de sources d'énergie renouvelable, ainsi que la consolidation du développement durable en Algérie.

\section{Conclusion}

L'utilisation des énergies renouvelables lutte contre l'effet de serre, en réduisant rejets de gaz carbonique dans l'atmosphère. En développement dans le monde entier, ces énergies permettent de gérer de façon intelligente les ressources locales et de créer des emplois.

L'Algérie à l'instar du reste du monde s'est lancée dans la production des énergies renouvelables en mettant en place un programme en deux phases, tel que mentionné ci-dessus.

\footnotetext{
${ }^{36} \mathrm{https}: / /$ www.energy.gov.dz/?article=resultats-attendus
} 
INTERNATIONAL JOURNAL OF

ADVANCED RESEARCH ON PLANNING AND

SUSTAINABLE DEVELOPMENT

Le potentiel national en énergies renouvelables étant fortement dominé par le solaire, la majorité des réalisations de la première phase concernait des centrales photovoltaïques.

Ce programme vise à valoriser les ressources nationales et à consolider le développement durable en Algérie, ainsi que le développement d'une industrie dans le domaine du renouvelable en tant que facteur de diversification de l'économie nationale.

\section{Bibliographie}

1. Futura planèt quels sont les cinq types d'énergies renouvelables? https://www.futura-sciences.com/planete/questions-reponses/energie-renouvelablesont-cinq-types-energies-renouvelables-4134/

2. Biosphère le point de vue des écologistes : https://biosphere.ouvaton.org/blog/lapopulation-mondiale-au-1er-janvier-2020/

3. Furura sciences, energie cinétique https://www.futurasciences.com/sciences/definitions/physique-energie-cinetique-9430/

4. Ministère de l'énergie https://www.energy.gov.dz/?rubrique=energies-nouvellesrenouvelables-et-maitrise-de-Irenergie

5. Cinq familles énergies renouvelables

http://www.energiesrenouvelables.org/media/photos/observer/5familles/5famillesER pdf

5. Algérie énergie renouvelable http://www.energy.gov.dz/francais/uploads/2016/Energie/energie-renouvelable.pdf 6. Énergies renouvelables : définition, exemples, avantages et limites (doc word) https://e-rse.net/definitions/energies-renouvelables-definition/\#gs.p4usk0

7. Les énergies renouvelables Questions- réponses http://www.enr.fr/userfiles/files/Brochures\%20G\%C3\%A9n\%C3\%A9rales/ENR\%20Q uestions-R\%C3\%A9ponses\%200K.pdf

8. Les energies renouvelables https://rs.umc.edu.dz/umc/Production Photoviltaique BF.pdf 
INTERNATIONAL JOURNAL OF

ADVANCED RESEARCH ON PLANNING AND

SUSTAINABLE DEVELOPMENT

9. Energies renouvelables développement et environnement discours, réalités et perspectives http://www.global-chance.org//MG/pdf/GC23.pdf

10. Le livre blanc des énergies renouvelables des choix qui fondent notre avenir http://www.smartgrids-cre.fr/media/documents/1202 SER LivreBlanc.pdf

11. Master les energies renouvelables

http://www.master-prnt.com/qse dd/pdf/2007/rapport energiesrenouvelables.pdf

12. Sources d'énergie renouvelable

file:///C:/Users/Dual\%20Computer/Desktop/sources d energie renouvelable.pdf

13. Planète healthy, énergie éolienne https://planetehealthy.com/energie-eolienne/

14. Éole https://fr.wikipedia.org/wiki/\%C3\%89ole

15. La petite histoire de l'énergie du vent http://energieavenirtpe.emonsite.com/pages/les-energies-renouvelables/l-energie-du-vent/la-petitehistoire-de-l-energie-du-vent.html

16. Les énergies renouvelables de la préhistoire à nos jourshttp://www.astrolabium.be//MG/pdf/dossier pe da e nergies.pdf

17. Accord de siège du centre régional pour les énergies renouvelables (2010) https://www.rcreee.org/sites/default/files/legal hostcountryagreement 2010 ar.pdf 18. Cairo Declaration of Intentions on Establishment of Regional Center for Renewable Energy and Energy Efficiency "RCREEE" June 25, 2008 https://www.rcreee.org/sites/default/files/legal cairodeclaration 2008 en.pdf

\section{Annexe : cadre législatif et règlementaire des énergies renouvelables en}

\section{Algérie}

1. Loi $n^{\circ} 14-10$ du 30 décembre 2014 portant loi de finances pour 2015 , notamment son article 108 qui prévoit la fusion des deux Fonds spéciaux « Le Fonds national pour la maîtrise de l'énergie (FNME) et le Fonds national pour les Energies renouvelables et la cogénération (FNER) » (JORA n`78 du 31 décembre 2014);

2. Loi $n^{\circ} 11-11$ du 18 juillet 2011 portant loi de finances complémentaire pour 2011, notamment son article 40 modifiant l'article 63 de la loi n 09-09 (JORA n40 du 20 juillet 2011) ; 
3. Loi n 09-09 du 30 décembre 2009 portant loi de finances pour 2010, notamment son article 64 portant création du fonds national pour les énergies renouvelables et la cogénération (FNER) (JORA n`78 du 31 décembre 2009);

4. Loi n 04-09 du14 août 2004 relative à la promotion des énergies renouvelables dans le cadre du développement durable (JORA n 52 du 18 août 2004) ;

5. Loi n $02-01$ du 05 février 2002 modifiée et complétée, relative à l'électricité et à la distribution du gaz par canalisations (JORA n॰ 08 du 06 février 2002) ;

6. Décret exécutif $n^{\circ} 17-98$ du 26 février 2017 définissant la procédure d'appel d'offres pour la production des énergies renouvelables ou de cogénération et leur intégration dans le système national d'approvisionnement en énergie électrique (JORA $n^{\circ} 15 \mathrm{du}$ 05 mars 2017) ;

7. Décret exécutif $n^{\circ} 16-121$ du 6 avril 2016 modifiant et complétant le décret exécutif $n^{\circ}$ 15-319 du 13 décembre 2015 fixant les modalités de fonctionnement du compte d'affectation spéciale $n^{\circ}$ 302-131 intitulé « Fonds national pour la maîtrise de l'énergie et pour les énergies renouvelables et de la cogénération »;

8. Décret exécutif $n^{\circ}$ 15-319 du 13 décembre 2015 fixant les modalités de fonctionnement du compte d'affectation spéciale n 302-131 intitulé « Fonds national pour la maîtrise de l'énergie et pour les énergies renouvelables et de la cogénération »;

9. Décret exécutif $n^{\circ} 15-69$ du 11 février 2015 fixant les modalités de certification de l'origine de l'énergie renouvelable et de l'usage de ces certificats ; (JORA n 09 du 18 février 2015) ;

10. Décret exécutif $n^{\circ}$ 13-218 du 18 juin 2013 fixant les conditions d'octroi des primes au titre des coûts de diversification de la production d'électricité. (JORA n 33 du 26 juin 2013) ;

11. Arrêté du 2 février 2014 fixant les tarifs d'achat garantis et les conditions de leur application pour l'électricité produite à partir des installations utilisant la filière éolienne (JORA n² 23 du 23 Avril 2014) ;

12. Arrêté du 2 février 2014 fixant les tarifs d'achat garantis et les conditions de leur application pour l'électricité produite à partir des installations utilisant la filière solaire photovoltaïque (JORA n² 23 du 23 Avril 2014) ; 
13. Arrêté du $1^{\text {er }}$ septembre 2014 fixant les tarifs d'achat garantis et les conditions de leur application pour l'électricité produite à partir des installations utilisant la filière de cogénération (JORA n 18 du 8 avril 2015) ;

14. Arrêté du 21 février 2008 fixant les règles techniques de raccordement au réseau de transport de l'électricité et les règles de conduite du système électrique (JORA $n^{\circ}$ 25 du 18 Mai 2008) ;

15. Arrêté interministériel du 19 avril 2008, portant Adoption du règlement technique relatif au «Module Photovoltaïque (PV) au silicium cristallin pour application terrestre». 